\title{
Experiencias y repercusión de una formación en ética de investigación
}

\author{
Carmen Rosa García Rupaya ${ }^{1}$
}

Resumen: El presente artículo tiene como propósito describir los logros y repercusiones de la capacitación en ética de la investigación que brinda el Centro Interdisciplinario de Estudios en Bioética de la Universidad de Chile, sirviendo de estímulo, motivación y orientación a profesionales que requieren conocer y aplicar las normas y el raciocinio conducente a la deliberación de los problemas en esta disciplina. Asimismo, describe cómo este conocimiento genera un efecto multiplicador en aspectos tales como la participación en un comité de ética de la investigación (CEI), organización de cursos y creación y desarrollo de líneas de investigación, que repercuten en publicaciones realizadas con estudiantes de posgrado. Relata además los contenidos y estrategias didácticas que pueden ser empleados en cursos de ética y bioética para estudiantes de estomatología y concluye mencionando la aplicación práctica de esta capacitación en los ámbitos docente, institucional y de investigación.

Palabras clave: ética, investigación, estomatología

\section{Experiences and impact of an ethics of research training}

Abstract: This article has the purpose to describe achievements and impact of the ethics of research training at the Interdisciplinary Center for Studies on Bioethics of the University of Chile, which has served as incentive, motivation and guide for professionals which require knowledge, regulations application and dialogue skills to reflect on problems of this discipline. Furthermore, it describes how this knowledge generates a multiplying effect in aspects such as participation in scientific ethical review committees, course organization and the creation and development of research lines, which generate publications by post degree students. It also narrates didactic contents and strategies which could be applied in ethics and bioethics courses for oral health students and concludes mentioning practical applications of this training for teaching, research and institutional development.

Key words: ethics, research, oral health

\section{Experiências e repercussão de uma formaçáo em ética da pesquisa}

Resumo: O presente artigo tem como propósito descrever os ganhos e as repercussóes da capacitação em ética da pesquisa oferecida pelo Centro Interdisciplinar de Estudos em Bioética da Universidade do Chile, que serve de estímulo, motivação e orientação para profissionais que necessitam conhecer e aplicar as normas e o raciocínio conducentes à deliberação dos problemas nesta disciplina. Assim, se descreve como este conhecimento gera um efeito multiplicador em aspectos tais como a participação num comitê de ética em pesquisa (CEP), organização de cursos, criação e desenvolvimento de linhas de pesquisa que repercutem em publicaçôes realizadas com estudantes de pós-graduação. Relata, ademais, os conteúdos e as estratégias didáticas que podem ser empregados em cursos de ética e bioética para estudantes de estomatologia e se conclui mencionando a aplicação prática desta capacitação nos âmbitos docente, institucional e de pesquisa.

Palavras-chave: ética, pesquisa, estomatologia

\footnotetext{
${ }^{1}$ Cirujano-Dentista UNFV, Perú. Diplomado Internacional Ética en Investigación Biomédica y Psicosocial, Egresada 2008. Fogarty Grant R25 TW006056-07

Correspondencia: crg_me@hotmail.com
} 


\section{Acerca del Programa de Formación en Ética de la Investigación Biomédica y Psicosocial}

El Centro Interdisciplinario de Estudios en Bioética (CIEB) de la Universidad de Chile convoca cada año a profesionales de América Latina a realizar estudios en el Programa de Formación en Ética de la Investigación Biomédica y Psicosocial, iniciativa apoyada por el Fogarty International Center-NIH, cuyo objetivo es capacitar en ética de investigación a personas que trabajan en el área en países en desarrollo(1).

Cada año hay una rigurosa selección de los participantes. Integré la quinta promoción en el periodo 2007-2008, cuyo grupo estuvo conformado por tres médicos y una enfermera que provenían de Colombia, Nicaragua, Bolivia, y yo misma, de profesión odontóloga en el Perú.

El curso incluye diversos tópicos relacionados con la ética y la investigación, pero lo interesante es el enfoque desde diversos ángulos. En tal sentido, permite entender los fundamentos de la bioética y, para su mejor comprensión, se analiza la implicancia del derecho y la antropología. Asimismo, se examinan los conflictos éticos en investigación biomédica y psicosocial, y en investigación con animales, todo esto a través de casos prácticos y publicaciones científicas(2-4).

Como es importante demostrar el conocimiento adquirido durante el periodo de formación, los becados deben elaborar un proyecto de investigación que ejecutan posteriormente en su país de origen; los cursos de metodología de la investigación, informática, búsqueda bibliográfica y estadística son herramientas esenciales para cumplir este objetivo.

El valor agregado, imposible de cuantificar por su gran trascendencia, es que la discusión y la retroalimentación de todos los conocimientos no solo incluye a profesores - de gran nivel- sino también a los mismos participantes(5), quienes poseen una amplia experiencia en sus instituciones. Asimismo, se suma el análisis crítico de estos temas de acuerdo con la problemática de su país de origen.

\section{Logros en capacitación en ética de la investi- gación}

Todo este preámbulo sirve de telon de fondo para analizar los logros personales obtenidos como resultado de esta capacitación en ética de la investigación, en diferentes ámbitos profesionales y académicos, sobre todo en mi labor como docente de pre y posgrado en estomatología.

Es así que en diciembre del 2007 fue posible realizar el Tercer Simposio Latinoamericano de Bioética en Odontoestomatología en la Facultad de Estomatología de la Universidad Peruana Cayetano Heredia (UPCH), en Lima, el cual congregó a expositores especialistas en el tema y sirvió para la reflexión y análisis de la formación bioética del cirujano dentista, con el objetivo de compartir la experiencia regional y promover con ello el desarrollo de capacidades, destrezas y habilidades que condujeran a mejorar la educación bioética en nuestra profesión(6).

Desde 2007, integrar el Comité Institucional de Ética en Investigación de la Universidad Peruana Cayetano Heredia ha reflejado la preparación recibida en el Programa, mediante un correcto y profundo análisis de los proyectos de investigación de pregrado, posgrado e instituciones externas que usualmente me designan revisar, obteniendo como resultado que la discusión y deliberación ética posterior a la presentación del proyecto es mejor sustentada.

\section{Curso de ética y bioética en pregrado}

Otro aspecto a resaltar es la incorporación, en 2009, del curso de ética y bioética en el plan de estudios de pregrado en estomatología en la universidad en la cual me desempeño como docente, siendo designada como coordinadora del curso. La formación adquirida en el CIEB permitió que organizara los contenidos del curso y aplicara estrategias didácticas que han generado motivación, interés y expectativa en los estudiantes de esta disciplina. El objetivo del curso es conocer y aplicar los fundamentos, principios y valores morales de la ética y bioética —necesarios como herramien- 
tas en la toma de decisiones frente a los diferentes problemas éticos/morales-durante la formación profesional en estomatología.

El curso estuvo organizado en su inicio en 2009 en cinco unidades didácticas y, posteriormente, a partir de 2010, en tres unidades didácticas²:

\section{Unidad didáctica 1}

Base conceptual ética y bioética.

Dignidad humana: Principio y final de vida.

- Principios de bioética. Poblaciones vulnerables.

- Conceptualización de moral, valores, ética, bioética y deontología.

- El valor fundamental de la dignidad humana. Declaración Universal de los Derechos $\mathrm{Hu}$ manos.

- Inicio de la vida, problemas éticos: clonación y reproducción asistida.

- Final de la vida, problemas éticos: limitación del esfuerzo terapéutico, eutanasia.

- Principios de la bioética.

- Fundamentación de la bioética latinoamericana: desde la bioética clínica a la bioética social.

- Poblaciones vulnerables. Factores asociados: culturales, institucionales, ambientales y del entorno, salud y educativos.

- Principios para superar la vulnerabilidad.

\section{Unidad didáctica 2}

Bioética en el campo odontológico. Alcances del Código de Ética del COP.

- Problemas éticos en el ejercicio de la profesión odontológica.

- Problemas bioéticos en el ejercicio de la profesión odontológica.

- Base legal y alcances del Código de Ética del COP.

- Contenido del consentimiento informado en la práctica clínica. Alcance legal.

\section{Unidad didáctica 3}

Ética en investigación científica.

Bioética y salud pública.

${ }^{2}$ Contenido del Curso Ética y Bioética, para estudiantes de Estomatología UPCH, elaborado por Mg. CD. Esp. Carmen Rosa García Rupaya.
- Código de Nuremberg, Declaración de Helsinki, Reporte Belmont, Normas CIOMS

- Legislación nacional.

- Función y alcances del Comité Institucional de Ética.

- Consentimiento informado en investigación biomédica.

El curso integra aspectos conceptuales y principios de la ética y la bioética, y sus enfoques en la odontología, en la investigación y en la salud pública. Incluye expositores invitados de distintas profesiones, porque es necesario que el alumno entienda que estad disciplinas son transversales a todas las profesiones. Propone que estas disciplinas deben generar un estilo de vida, tanto en el ámbito personal como profesional. Este mensaje se transmite a través de diversas estrategias didácticas, como clases magistrales, talleres, estudios de caso, exposiciones y visionado crítico; esta última estrategia ha sido la que ha generado más interés en los estudiantes, porque, con fines didácticos, mediante la revisión de fragmentos específicos de películas de cine y televisión, los alumnos analizan y discuten los diversos conflictos éticos planteados. Esta estrategia fue implementada porque el público al cual va dirigido el curso son estudiantes bastante jóvenes, entre 17 y 20 años, por lo que era necesario que las actividades prácticas tuvieran carácter interactivo.

Se pretende orientar el análisis hacia la reflexión de los dilemas que puede enfrentar el estudiante en el desarrollo de su vida profesional, resaltando la necesidad de interiorizar valores de responsabilidad, honestidad y justicia como primordiales en la relación odontólogo-paciente(7).

Ha sido de mi interés que la formación en ética de la investigación se difunda convenientemente. En ese sentido, obtuve que el CIEB de la Universidad de Chile, mediante un convenio, implementara una pasantía para estudiantes de maestría de la UPCH por el lapso de una semana, siendo de gran importancia esta interacción con los profesionales becarios del Programa, debido a que el magíster debe formarse básicamente para realizar investigación y es fundamental que adquiera competencias para la aplicación de las normas y 
principios éticos en investigación en seres humanos y animales.

\section{Línea de investigación en aspectos éticos y bio- éticos}

La formación ética en el Programa del Cieb ha repercutido también en generar una línea de investigación, la cual se va fortaleciendo constantemente por el trabajo que se realiza con los estudiantes de maestría. Los principales tópicos estudiados son: conocimientos y actitudes en ética y bioética en el ámbito de alumnos y docentes en estomatología; el proceso de consentimiento informado desde la visión del odontólogo y del paciente; la aplicación de consideraciones éticas en la investigación científica en pre y posgrado, entre otros.

Para medir el nivel de conocimiento en ética y bioética en estudiantes y docentes de odontología, se elaboró un cuestionario - validado mediante un juicio de expertos - y una prueba piloto para medir su confiabilidad y reproducibilidad(8). Posteriormente, fue aplicado en otra población de estudiantes de odontología en la zona central del país. Se obtuvieron resultados muy interesantes que denotan conocimientos en estas disciplinas en ambos grupos(9).

Otra investigación se orientó a identificar el proceso del consentimiento informado desde el punto de vista del paciente, siendo este un tema poco estudiado en odontología. Para ello se elaboró un instrumento con cuatro dimensiones: información, entendimiento, valores del profesional y autonomía. La intención era obtener la apreciación del paciente acerca de cómo se realiza este proceso, encontrándose resultados favorables y concluyendo que se debe insistir en utilizar materiales educativos que permitan una mejor comprensión del tipo de tratamiento que realizará el especialista(10).

Asimismo, resultó interesante estudiar, revisando los trabajos de tesis de la última década, cómo se vienen aplicando las consideraciones éticas en las investigaciones que se realizan en pre y posgrado en estomatología. Los resultados mostraron que en los últimos cinco años, y sobre todo en el nivel de maestría, se presentan estos aspectos más claramente redactados y con una mayor presencia(11).

En conclusión, la formación en ética de investigación brindada por el CIEB, bajo el auspicio del Fogarty International Center, fue para mí una experiencia de incorporación de conocimientos y habilidades que pude reflejar posteriormente en forma práctica en los ámbitos docente e institucional, así como en el comité de ética y en la investigación en esta disciplina. La formación recibida tuvo su expresión en los diversos aspectos reseńados, pero sobre todo en la mirada ética y bioética que imprime una nueva manera de situarse personal y profesionalmente en la odontoestomatología y en la relación médico-paciente. 


\section{Referencias}

1. Vicerrectoría de Investigación y Desarrollo. Centro Interdisciplinario de Estudios en Bioética. Universidad de Chile. Programa de Formación en Ética de la Investigación Biomédica y Psicosocial. (Citado 02/02/2012). Disponible en: http://www.bioetica.uchile.cl/cursos/postulacion.htm

2. Lolas F, Quezada A, Rodriguez E. (eds.) Investigación en Salud. Dimensión Ética. Santiago de Chile: CIEB, Universidad de Chile; 2006.

3. Cardozo C, Lolas F, Rodríguez E, Quezada A. Ética y Odontología. Una Introducción. Santiago de Chile: CIEB, Universidad de Chile; 2007.

4. Lolas F. Ética e Innovación Tecnológica. Santiago de Chile: CIEB, Universidad de Chile; 2006.

5. Acuerdo de Colaboración CIEB Universidad de Chile, Programa de Bioética OPS. Informe de Actividades 2007-2008. (Citado 02/02/2012) Disponible en: http://www.bioetica.uchile.cl/lectura/selec.htm

6. Bioética Informa 2007; 12(44).

7. García-Rupaya C. Inclusión de la ética y bioética en la formación de pre y posgrado del cirujanodentista en Perú. Acta Bioethica 2008; 14(1): 74-77.

8. Rodríguez-Villegas M. Nivel de conocimiento en bioética en la práctica odontológica cotidiana de los estudiantes de tercer y quinto año de la Facultad de Odontología de la Universidad Católica Santa María Arequipa, Perú 2007. Tesis de Maestría en Estomatología. Universidad Peruana Cayetano Heredia.

9. Samaniego-Napayco O. Nivel de conocimientos en ética y bioética en docentes y estudiantes de odontología de la Universidad Peruana Los Andes, 2010. Tesis de Maestría en Estomatología. Universidad Peruana Cayetano Heredia.

10. Alfaro-Carballido LD, García-Rupaya CR. Percepción del proceso de consentimiento informado en pacientes de la Clínica Estomatológica Central de la Universidad Peruana Cayetano Heredia. Rev Estomatol Herediana 2011; 21(1): 5-12.

11. Cáceda-Gabancho K, García-Rupaya CR. Aplicación de consideraciones bioéticas en investigación científica en estomatología en pre y posgrado de la Universidad Peruana Cayetano Heredia en los últimos 10 años. Rev Estomatol Herediana 2011; 21(2): 73-78.

Recibido: 20 de febrero de 2012

Aceptado: 27 de marzo de 2012 